\title{
Primary gastric cancer presenting with a metastatic embolus in the common carotid artery: a case report
}

\author{
Ying Zhang ${ }^{1}$, Xiu-feng Zhang ${ }^{2}$, Wei-hui Shentu', Guan-gen Yang ${ }^{2}$, Zhong Shen ${ }^{2}$ and Pin-tong Huang ${ }^{1 *}$
}

\begin{abstract}
Although about $30 \%$ of gastric cancers have distant metastasis at the time of initial diagnosis, metastatic tumor embolus in the main blood vessels is not common, especially in the main artery. The report presents, for the first time, an extremely rare clinical case of a metastatic embolus in the common carotid artery (CCA) from primary gastric cancer. Metastatic embolus from the primary tumor should be considered when patients present with gastric cancer accompanied by intravascular emboli. The patient should be actively examined further so as to allow early detection and treatment.
\end{abstract}

Keywords: Tumor embolus, Common carotid artery, Metastasis, Gastric cancer

\section{Background}

Metastatic tumor embolus in the common carotid artery from primary gastric cancer is an exceedingly rare event. Here, we report on the case of a 69-year-old male with primary gastric cancer presenting with a metastatic tumor embolus in the common carotid artery who underwent a palliative subtotal gastrectomy and endarterectomy, which is the first report of metastatic embolus in the common carotid artery in English and non-English literature.

\section{Case presentation}

The patient was a 69-year-old male patient who had been hospitalized for four days with sudden left limb weakness. Four days previously, during outdoor activities, this patient had felt sudden left limb weakness accompanied by instability in holding materials with his left arm, unsteady gait of his left leg and impaired speech articulation. Physical examination revealed: conscious, heart and lung (-); neurological symptoms: isocoria, light reflex was normal, mouth slightly favored the right, the left nasolabial fold was a little shallow, tongue lolled to the left side,

\footnotetext{
* Correspondence: huangpintong@126.com

'Department of Ultrasound, The Second Affiliated Hospital, Zhejiang University College of Medicine, No.88 Jiefang Road, Hangzhou, Zhejiang 310009, China

Full list of author information is available at the end of the article
}

left side paresis test $(+)$, Pakistan's sign of the left side $( \pm)$, Pakistan's sign on the right side (-), limb muscle strength and muscular tension was normal. Emergency cranial computed tomography (CT) scan in another hospital showed that hypodense lesions in the right basal ganglia were detected and the diagnosis of cerebral infarction was considered (Figure 1). In our hospital, magnetic resonance imaging (MRI) examination revealed right basal ganglia cerebral infarction and ischemic lesions (Figures 2-A.B). Carbohydrate antigen (CA) 19-9: 12.35U/ml, carcinoembrionic antigen (CEA): $25.28 \mathrm{ng} / \mathrm{ml}$, CA72-4: $21.73 \mathrm{U} / \mathrm{ml}$. The patient denied having 'hepatitis, tuberculosis, diabetes, hypertension, coronary heart disease' and gave a family genetic history. He related a history of 'chronic gastritis' for the last three years, and having occasional upper abdominal discomfort after meals for the last six months. His brothers and sisters also had history of gastritis.

Conventional carotid ultrasonography showed that the diameters of the bilateral common carotid artery were normal. The intima-media thickness (IMT) of the left side was $0.8 \mathrm{~mm}$. A hypoechoic mass with an irregular contour was observed at the right common carotid artery. The size of the mass was about $29 \times 7 \mathrm{~mm}$, with heteroechogenicity. A pedicled structure, which connected the mass with the common carotid artery wall, could be seen and the mass could swing slightly following carotid pulsation (Figure 3).

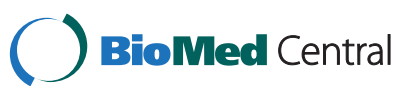




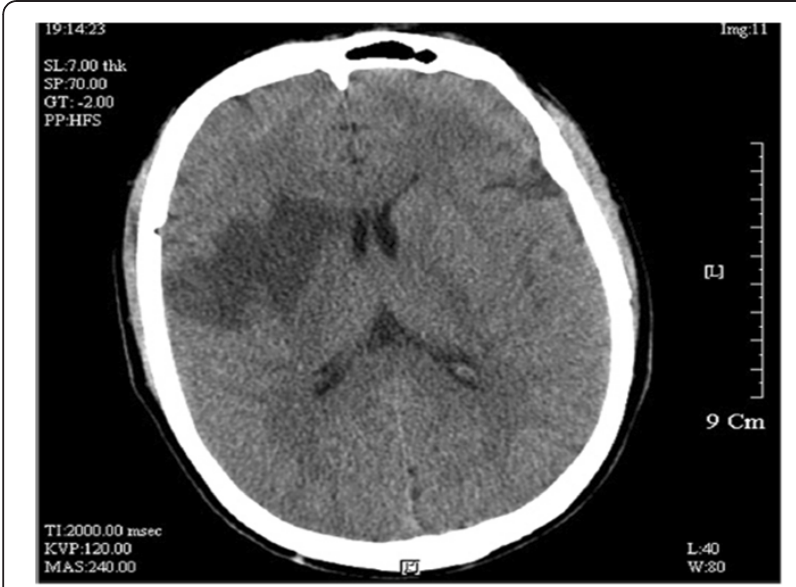

Figure $1 \mathrm{CT}$ showed hypodense lesions in the right basal ganglia.

The contrast-enhanced ultrasonography (CEUS) was then performed and displayed the mass located on the posterior common carotid artery wall. About 14 seconds after the injecting of the contrast agent, the carotid arterial lumen enhanced first. About 18 seconds after the injecting of the contrast agent, the visible point-like enhancement within the hypoechoic mass could be seen. At approximately 32 seconds, the enhancement of the mass reached the peak, and began to wash out at about 48 seconds (Figure 4 ).

Considering the 'three-year history of chronic gastritis', an abdominal ultrasound examination was routinely performed and revealed a thickened gastric antrum wall. Further double contrast-enhanced ultrasonography (DCEUS) showed that the wall of the gastric angle and

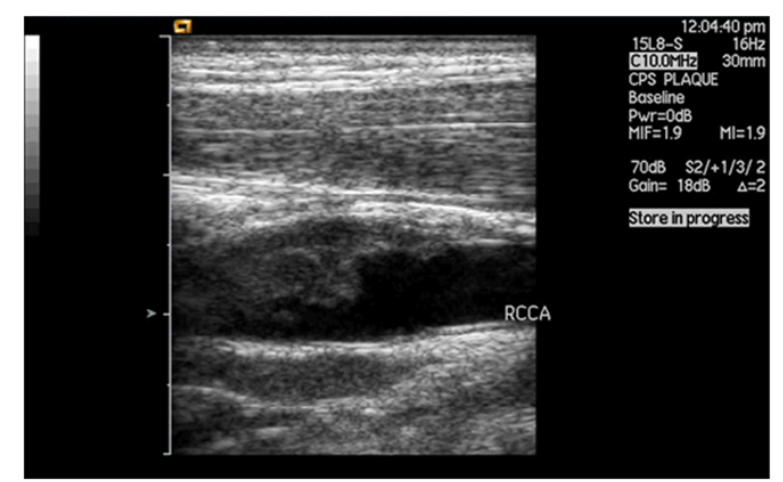

Figure 3 Carotid ultrasonography showed that the diameters of bilateral common carotid artery were normal. A hypoechoic mass with irregular contour was observed at the right common carotid artery. The size of the mass was about $29 \times 7 \mathrm{~mm}$, with heteroechogenicity. antrum were obviously thickened with hypoechoic inner echogenicity. Five layers of normal gastric wall had disappeared; the basal boundary was obscure. The hypoechoic lesion involved all layers of the gastric wall. Enlarged intra-abdominal lymph nodes with clear borders were detected around the gastric pylorus. The size of the largest lymph node was about $10 \times 8 \mathrm{~mm}$.

The preliminary impression from the ultrasound was advanced gastric cancer with carotid arterial tumor embolus, which was confirmed by the biopsy from the gastroscope and specimens obtained from the palliative, subtotal gastrectomy and endarterectomy. The poorly differentiated adenocarcinoma and partial signet-ring cell carcinoma in the gastric antrum was confirmed by histopathological examination (Figures 5, 6).
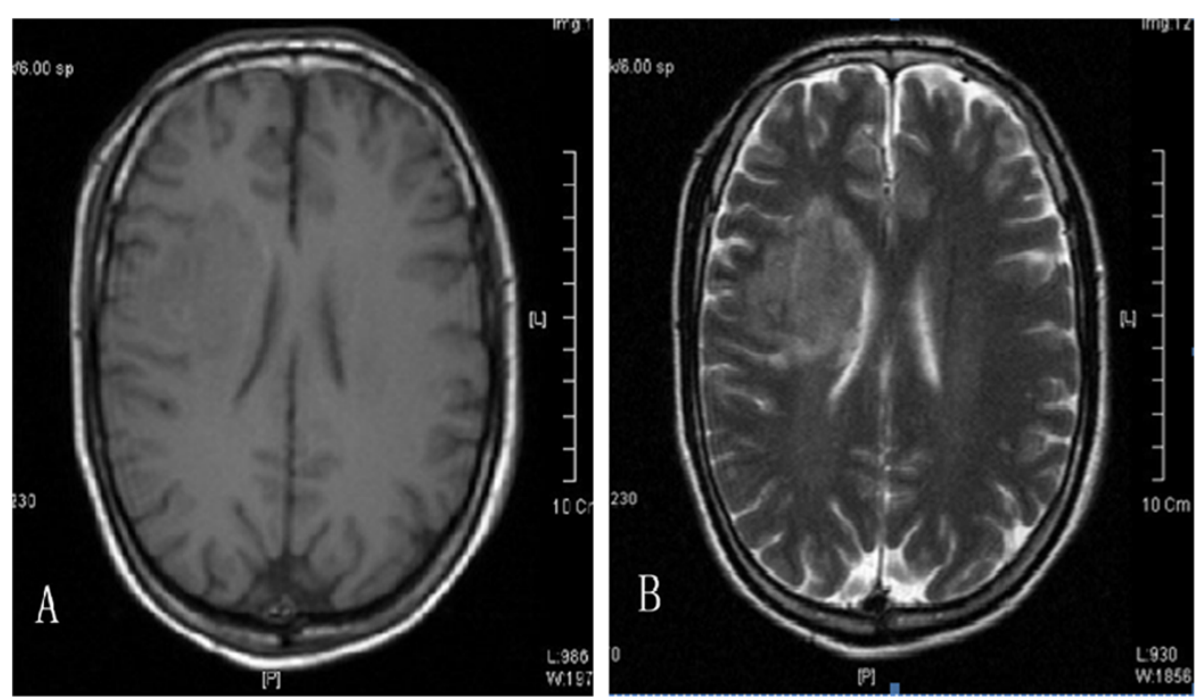

Figure 2 A. MRI (T1-weighted image) showed right basal ganglia cerebral ischemic lesions. B. MRI (T2-weighted image) showed right basal ganglia cerebral ischemic lesions. 


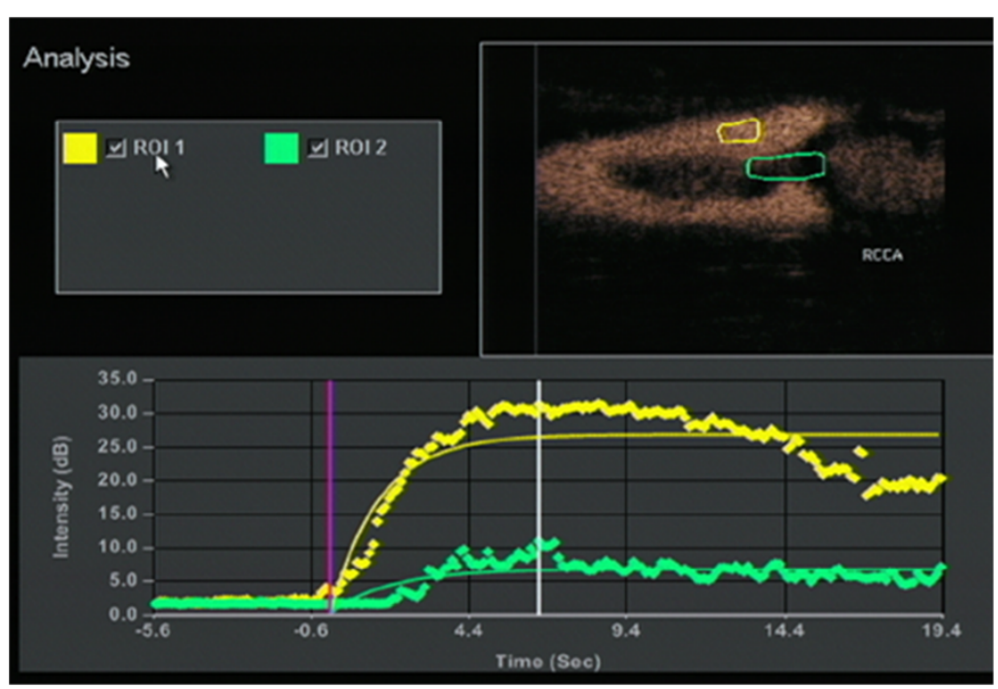

Figure 4 Time-intensity curve analysis in two user-defined regions of interest within the common carotid artery (yellow curves) and the lumen of the hypodensity lesions (green curves).

\section{Discussion}

The routine carotid ultrasonography was performed because of the occurrence of the cerebral embolism. The tumor embolus in the common carotid artery was subsequently found and showed a hypoechoic mass with an uneven surface at the right common carotid artery bifurcation. It was different from the common carotid atherosclerotic plaque. The imaging features of carotid plaque in ultrasound examinations have been reported in many published works [1-3]. Considering the history, a middle-aged patient, with no obvious thickening of the common carotid artery intima, without hypertension, diabetes, coronary heart disease and other risk factors for cerebrovascular disease, and the characteristics of the carotid ultrasonography: a hypoechoic mass with a pedicle, the typical 'fast-in, fast-out' pattern which is not consistent with a thrombus and atherosclerotic plaque,

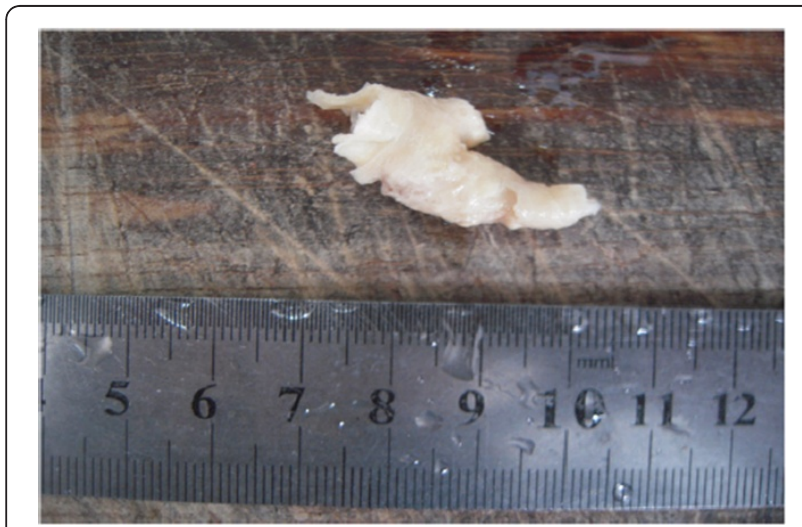

Figure 5 The gross specimen of metastatic tumor embolus in the common carotid artery after endarterectomy. the hypoechoic mass was suspected to be a tumor embolus. Given the complication of the history of chronic gastritis, abdominal ultrasound was ordered and found that there was nonuniform thickening of the gastric antrum wall. DCEUS has been widely used for diagnosis of gastric cancer in China [4]. It can be used in the preoperative staging of gastric cancer and has been widely applied in clinics. Many published works have reported on this method, which combines an ultrasonic oral contrast agent and a bolus injection of SonoVue to display the gastric wall. The method can help us to observe features of thickening and perfusion type of the gastric wall. The results of the DCEUS of this patient showed diffuse nonuniform thickening of the gastric wall, the

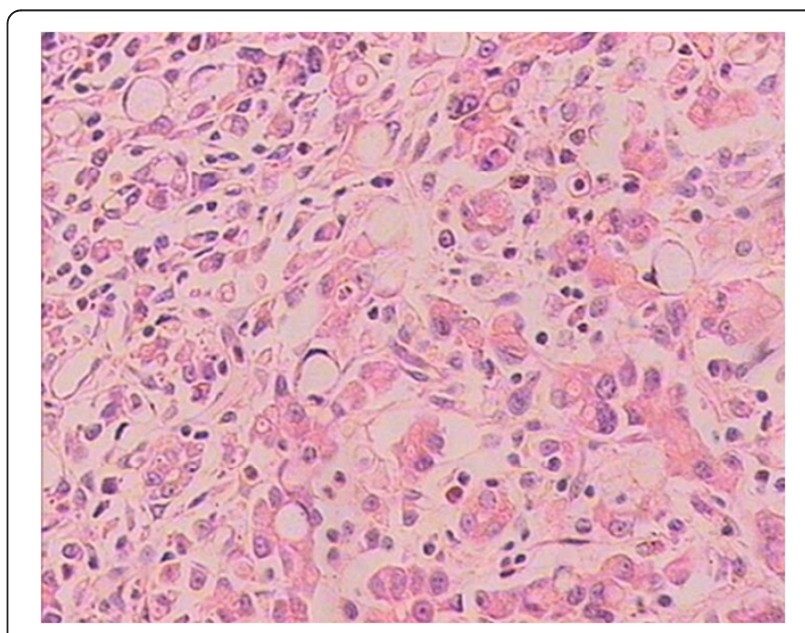

Figure 6 Histological findings with H\&E staining of embolus in the common carotid artery showed the poorly differentiated adenocarcinoma and partial signet-ring cell carcinoma $(\times 400)$. 
lesion penetrated the serosa, there was a fast perfusion pattern, and several lymph nodes could be seen around abdominal aorta. The results of the DCEUS were consistent with the gastroscope: advanced gastric cancer. Lymph node and hematogenous metastasis (especially in the portal vein) are common pathways in advanced gastric cancer [5]. Considering the common carotid artery tumor embolus had metastasized from the advanced gastric cancer, we corrected the original diagnosis.

\section{Conclusion}

This report presented, for the first time, an extremely rare case of a metastatic tumor embolus in the common carotid artery from primary gastric cancer. The case described was a clinical entity that can be easily misdiagnosed as atheromatous plaque, thrombus, and so on. Metastatic embolus from the primary tumor should be considered in patients with gastric cancer accompanied by intravascular emboli, and patients should be actively examined further so as to allow early detection and treatment.

\section{Consent}

Written informed consent was obtained from the patient for publication of this case report and any accompanying images.

\author{
Abbreviations \\ CCA: Common carotid artery; CEUS: Contrast-enhanced ultrasonography; \\ CT: Computed tomography; DCEUS: Double contrast-enhanced \\ ultrasonography; IMT: Intima-media thickness; MRI: Magnetic resonance \\ imaging.
}

\section{Competing interests}

The authors declare that they have no competing interests.

\section{Authors' contributions}

YZ performed the literature review, drafted and co-wrote the manuscript with XFZ. GGY and ZS revised the manuscript for intellectual content. WHST and PTH evaluated the ultrasound features and contributed to the ultrasound part of manuscript. All authors have read and approved the final version of the manuscript.

\section{Authors' information}

Xiu-feng Zhang was the co-first author.

\section{Acknowledgements}

This work was supported by grants from the National Natural Science Foundation of China (No. 81071164, No. 81271584, and No. 81101876) and the Key Specialty and Special Disease Foundation of Hangzhou Science and Technology Bureau (Number 20100733Q12,Number 20110733Q01).

\section{Author details}

'Department of Ultrasound, The Second Affiliated Hospital, Zhejiang University College of Medicine, No.88 Jiefang Road, Hangzhou, Zhejiang 310009, China. ${ }^{2}$ Department of Colorectal Surgery, The Third People's Hospital of Hangzhou, No. 38 Westlake Avenue, Hangzhou, Zhejiang 310009, China.

Received: 5 July 2012 Accepted: 14 October 2012

Published: 30 October 2012

\section{References}

1. Acharya UR, Sree SV, Krishnan MM, Molinari F, Saba L, Ho SY, Ahuja AT, Ho SC, Nicolaides A, Suri JS: Atherosclerotic risk stratification strategy for carotid arteries using texture-based features. Ultrasound Med Biol 2012, 38:899-915.

2. Funaki T, lihara K, Miyamoto S, Nagatsuka K, Hishikawa T, Ishibashi-Ueda H: Histologic characterization of mobile and nonmobile carotid plaques detected with ultrasound imaging. J Vasc Surg 2011, 53:977-983.

3. Faggioli GL, Pini R, Mauro R, Pasquinelli G, Fittipaldi S, Freyrie A, Serra C, Stella A: Identification of carotid 'vulnerable plaque' by contrastenhanced ultrasonography: correlation with plaque histology, symptoms and cerebral computed tomography. Eur J Vasc Endovasc Surg 2011, 41:238-248.

4. Huang P, Li S, Aronow WS, Wang Z, Nair CK, Xue N, Shen X, Chen C Cosgrove D: Double contrast-enhanced ultrasonography evaluation of preoperative Lauren classification of advanced gastric carcinoma. Arch Med Sci 2011, 7:287-293.

5. Asaumi Y, Miyanaga T, Ito H, Shin H, Fujita M, Miyazaki M, Yagi D, Kitamura $H$, Hirano M, Hayashida Y, Maeda K, Ohta K, Hayashi H, Doden K, Hattori M, Hashizume $Y$, Kaizaki $Y$ : A case of advanced gastric cancer with tumor embolus in the portal vein successfully treated with S-1 and CDDP therapy. Gan To Kagaku Ryoho 2011, 38:1175-1178.

\section{doi:10.1186/1477-7819-10-229}

Cite this article as: Zhang et al:: Primary gastric cancer presenting with a metastatic embolus in the common carotid artery: a case report. World Journal of Surgical Oncology 2012 10:229.

\section{Submit your next manuscript to BioMed Central and take full advantage of:}

- Convenient online submission

- Thorough peer review

- No space constraints or color figure charges

- Immediate publication on acceptance

- Inclusion in PubMed, CAS, Scopus and Google Scholar

- Research which is freely available for redistribution 